\title{
Caractère «porteur de la flavescence dorée " chez les vignes porte-greffes, en particulier le 3309 Couderc et le Fercal
}

\author{
A Caudwell ${ }^{1}, \mathrm{~J}_{\text {Larrue }}{ }^{*}, \mathrm{~V}$ Tassart $^{2}$ \\ avec la collaboration de $\mathrm{R}$ Boidron $^{2}, \mathrm{~S}$ Grenan $^{2}, \mathrm{M}$ Leguay $^{3}, \mathrm{P}$ Bernard $^{4}$ \\ 1 INRA, station de recherches sur les mycoplasmes et arbovirus des plantes, BV 1540, F21034 Dijon cedex; \\ 2 Établissement national technique pour l'amélioration de la viticulture, domaine de l'Espiguette, \\ F30240 Le Grau-du-Roi, \\ 3 Office national interprofessionnel des vins, 232, rue de Rivoli, F75001 Paris; \\ ${ }^{4}$ Service de la protection des végétaux, chemin de la Jasso, F11000 Carcassonne, France
}

(Reçu le 21 avril 1993 ; accepté le 8 décembre 1993)

\begin{abstract}
Résumé - La plupart des variétés de porte-greffes de vigne peuvent être porteuses sans symptôme de la flavescence dorée (FD). Ces ceps infectés peuvent être reconnus, dans une certaine mesure, chez le $3309 \mathrm{C}$ et le Fercal, par un retard printanier de végétation et le mauvais aoûtement de certains rameaux. Une fois infectés, il apparaît que ces ceps restent porteurs toute leur vie. Par conséquent, au contraire de ce qui se passe pour les variétés de $V$ vinifera, les traitements aux insecticides pour éviter les inoculations par l'insecte vecteur ne sont pas efficaces pour réduire l'infectivité des plantes déjà infectées. Ces porte-greffes ne transmettent la maladie que dans une proportion de leurs boutures variant de 6 à $80 \%$ selon les ceps. Les rameaux d'un même cep sont inégalement infectés et le niveau d'infection, le long du rameau, apparaît fortuit. La forte transmission de la FD par les boutures infectées de $3309 \mathrm{C}$ est associée à une forte mortalité des greffes, la «mortalité pratique (greffes mortes + malades) pouvant atteindre $82 \%$. De nouvelles inoculations décelées dans un champ de pieds mères traité aux insecticides soulignent la nécessité d'un bon isolement géographique des vignes mères.
\end{abstract}

vigne / flavescence dorée / porte-greffes / MLO / sélection sanitaire

Summary - Ability of grapevine rootstocks varieties to transmit flavescence dorée. Study of the case of $3309 \mathrm{C}$ and Fercal. Most grapevine rootstock varieties are potential symptomless carriers of Flavescence dorée (FD). However some infected plants of $3309 \mathrm{C}$ and Fercal rootstocks can be recognized as they exhibit late spring growth and incomplete ripening of some canes. Once infected, these plants remain as potential sources of infection throughout their lifetime. Therefore, contrary to $V$ vinifera behaviour, treating fields with insecticides against the leafhopper vector to prevent inoculations of the disease is not efficient in reducing the infectivity of the plants already infected. Only $6-80 \%$ of cuttings obtained from infected plants transmit the disease. Canes from the same vine are unevenly infected and the infection level along the canes is also uneven. The high degree of $F D$ transmission in $3309 \mathrm{C}$ is associated with a high graft mortality, the 'practical mortality' (graft death + diseased) sometimes reaching $82 \%$. New inoculations can occur in fields containing 3309 mother plants, even though insecticide treatments are mandatory. This stresses the necessity of a geographical isolation of mother-plant stands.

grapevine / flavescence dorée / rootstock / MLO / certification

\footnotetext{
* Correspondance et tirés à part.
} 


\section{INTRODUCTION}

Le comportement des variétés cultivées de $\mathrm{Vi}$ tis vinifera atteintes de flavescence dorée (FD) est assez bien connu. II a fait l'objet de plusieurs études scientifiques et pratiques. Nous savons que presque toutes les variétés sont sensibles à des degrés divers. La plupart se rétablissent à la suite des premiers symptômes, qui sont généralement graves et généralisés (crise). Ce rétablissement est définitif si la plante n'est pas réinoculée. C'est le cas des vignes où sont effectués des traitements insecticides contre la cicadelle vectrice Scaphoideus titanus Ball. Le rétablissement s'accompagne d'une certaine résistance de la plante (notable pendant les 3 ans qui suivent) et les réinoculations de la maladie aboutissent à des symptômes qui restent localisés à quelques rameaux autour du point d'inoculation (Caudwell, 1964). Certaines variétés, telles que le Nielluccio (Sangiovese), ne se rétablissent pas; les symptômes persistent plusieurs années et conduisent à la mort de la plante (Caudwell et al, 1974). Il existe, selon les variétés, tous les intermédiaires entre ces 2 comportements.

Les portes-greffes avaient été peu étudiés parce qu'ils ne montrent que peu de symptômes ou aucun symptôme. Certains réagissent lorsqu'ils sont inoculés en pépinière (Moutous, 1977). Des repousses présentant des symptômes ont été observées occasionnellement sur plusieurs variétés. Ainsi, Vitis rupestris cv du Lot a été reconnu malade, mais survivant sur les pieds de Nielluccio morts de la FD en Corse (Caudwell et al, 1974). II montrait des feuilles uniformément jaunes et repliées en cuillère vers le dessus. Cette espèce est donc porteuse et relativement sensible à cette maladie (fig 1). La manifestation des symptômes de la FD sur le porte-greffe $\mathrm{SO} 4$ a été observée sur les repousses d'une parcelle très malade de Castelnaudary (Aude). Aucun symptôme n'a été relevé, à l'exception d'un seul cep sur lequel ce porte-greffe présentait un non-aoûtement sur un rameau, accompagné d'une légère décoloration du limbe avec enroulement (fig 2). Ces 2 symptômes participent au syndrome habituel de la FD, reconnu sur les variétés de Vitis vinifera. C'est le signe que le SO4 serait également porteur potentiel de la maladie (Banton, Larrue et Caudwell, communication personnelle).

Les repousses du porte-greffe 1103 Paulsen ont été observées sur une vigne d'Ali- cante Bouschet très malade dans les Corbières (Aude). La plupart de ces repousses ne montraient aucun symptôme. Cependant, ici et là, sur environ $8 \%$ des ceps, on a pu relever quelques non-aoûtements des bois de ce porte-greffe. Le viticulteur a fait, de sa propre initiative, quelques regreffages en Alicante Bouschet sur ces porte-greffes. Ces regreffages sont immédiatement devenus malades. Le $1103 \mathrm{P}$ doit donc être considéré comme porteur potentiel de la FD, même lorsqu'il ne montre aucun symptôme (Tassart, communication personnelle).

Un cep de $420 \mathrm{~A}$ a été repéré dans l'Aude durant l'été 1990 avec des symptômes de FD voisins de ceux que l'on peut observer sur les variétés de $V$ vinifera: bois non-aoûtés, feuilles enroulées et gaufrées. L'indexage par greffage en vert a confirmé qu'il était porteur de la FD (Tassart, communication personnelle).

À quelques exceptions près, les variétés de porte-greffes ne montrent pas de symptôme bien caractérisé de la FD, et il est nécessaire de savoir si elles sont porteuses de la maladie ou non. II importe d'étudier si elles se rétablissent ou si elles sont porteuses définitives. Enfin, il est urgent d'évaluer le danger de la propagation de la FD par les bois des porte-greffes.

Ces problèmes doivent être étudiés pour les 20 variétés de porte-greffes les plus utilisées en France. Ils ont été abordés selon plusieurs protocoles. Nous voudrions exposer ici les résultats obtenus par l'étude de champs de pieds mères expérimentaux. Nous analyserons les signes de maladie, même les plus discrets, qui ont pu être relevés, les résultats des indexages, la répartition des boutures infectieuses le long des rameaux du pied mère, et l'évolution pluriannuelle des descendances des pieds mères infectés.

Ce travail a débuté sur la variété de portegreffe $3309 \mathrm{C}$. II a été étendu au Fercal et, avec moins de succès, à quelques autres variétés.

\section{MATÉRIEL ET MÉTHODES}

Les champs de pieds mères expérimentaux qui font l'objet de cette étude sont situés dans l'Aude, au centre des zones touchées par l'épidémie de FD. Ils sont constitués de pieds taillés en tête de saule au ras du sol et conduits sans palissage, en laissant courir les rameaux sur le sol, selon la technique en usage dans le sud de la France. 
II s'agit de plantations clonales, sensées être constituées au départ de plants identiques au point de vue génétique et sanitaire. Des hétérogénéités peuvent apparaître par la suite, du fait des aléas propres à tout vignoble. Plantées vers la fin des années 1970, elles ont subi l'épidémie de FD entre 1982 et 1986. Depuis 1987, elles ont été traitées aux insecticides selon les recommandations officielles, propres aux champs de pieds mères. On peut considérer les ceps malades de ces plantations comme à peu près homogènes à partir de 1989, date du début de notre expérimentation.

La première étape a été l'observation et le suivi plusieurs fois par an pour relever toute anomalie pouvant être rapportée à la FD. Un classement des symptômes a été tenté selon ces premiers résultats. Les pieds suspects ont été indexés avec une variété sensible, le Chardonnay en général (fig 3). Lorsque d'autres indicateurs ont été utilisés, tels le Pinot noir ou le Baco $22 \mathrm{~A}$, nous le signalerons dans le tableau des résultats. Les indexages faits en greffes d'hiver sur table ont été refaits en greffe en vert pour le Fercal qui n'a pas eu une bonne reprise en greffe d'hiver. Régulièrement, certains des indexages positifs ont été testés par l'épreuve sérologique Elisa (Caudwell et Kuszala, 1992) qui a montré que dans tous les cas, il s'agissait de la FD sensu stricto.

Nous avons choisi de présenter les résultats pluriannuels en regroupant les ceps selon l'année où les premiers "symptômes" ont été observés. Cette méthode a l'avantage de réunir les ceps qui ont fait l'objet du même nombre d'années de notations et d'indexages et de faire ressortir les anomalies d'évolution telles que d'éventuelles réinoculations.

La deuxième étape a été de repérer la localisation sur le cep des zones infectieuses, de façon à contrôler les risques de propagation de la maladie par les bois et de guider le choix des échantillons pour les indexages et les tests sérologiques. Cette étude a été menée en plantant les boutures indexées en greffes d'hiver dans l'ordre où elles se trouvaient sur le rameau du pied-mère. L'étendue des zones infectieuses a été calculée en nombre de boutures consécutives indexées FD, chaque bouture étant estimée d'une longueur de $30 \mathrm{~cm}$.

\section{RÉSULTATS}

\section{Étude du champ de pied mère expérimental de 3309}

Les signes discrets de FD qui ont été décrits en introduction sur des repousses de portegreffes de ceps atteints de FD ont conduit à étudier la présence de la FD directement dans les champs de pieds mères de portegreffes. Nous avons choisi d'aborder ce travail dans une vigne expérimentale de $3309 \mathrm{C}$ située dans l'Aude, dans la zone où s'est développée l'épidémie de FD.

Aucun symptôme typique de la FD n'a été reconnu, mais certaines plantes ont montré une croissante lente au printemps. Plus tard, au moment où les cercles de végétation des différents ceps commencent à se recouper, ce retard de végétation s'est estompé, mais certaines de ces plantes ont révélé à l'automne un mauvais aoûtement sur un ou plusieurs rameaux. Ces 2 caractères, retard de végétation et non-aoûtement partiel, entrent dans le syndrome de la FD tel qu'il est connu sur les variétés de $V$ vinifera, mais ils sont peu spécifiques en comparaison d'autres caractères de la FD.

De ces premiers résultats, 3 classes de plantes, $A, B$ et $C$, ont pu être différenciées selon la première note $A, B$, ou $C$ qui leur $a$ été donnée :

- note A : plantes montrant un retard de végétation au printemps et quelques signes de mauvais aoûtement en automne ;

- note B : plantes montrant un retard de végétation au printemps, mais aucun symptôme de non-aoûtement ;

- note $\mathrm{C}$ : plantes ne montrant aucun symptôme, mais situées au voisinage immédiat des plantes de classe $A$. On sait en effet que la propagation naturelle de la FD se fait en taches denses au centre, plus dispersées sur la périphérie (Caudwell, 1964).

Incidemment, certains ceps de classe A ont pu montrer un léger jaunissement des feuilles, accompagné d'un discret enroulement vers le dessus de la feuille, à la manière de $V$ rupestris cité plus haut. Parfois, des décolorations de feuille limitées par les principales nervures ont pu se manifester, mais ces caractères sont apparus trop rarement pour être retenus dans la définition des classes de symptômes.

Chacune des plantes repérées de classe $A, B$ ou $C$ a fait l'objet d'une notation et d'un indexage annuel avec une variété sensible, Chardonnay ou Baco 22 A (fig 3).

Les résultats sont présentés en regroupant les ceps selon leur classe de symptôme A, $B$ ou $C$ et selon l'année où ils ont été ainsi repérés. 

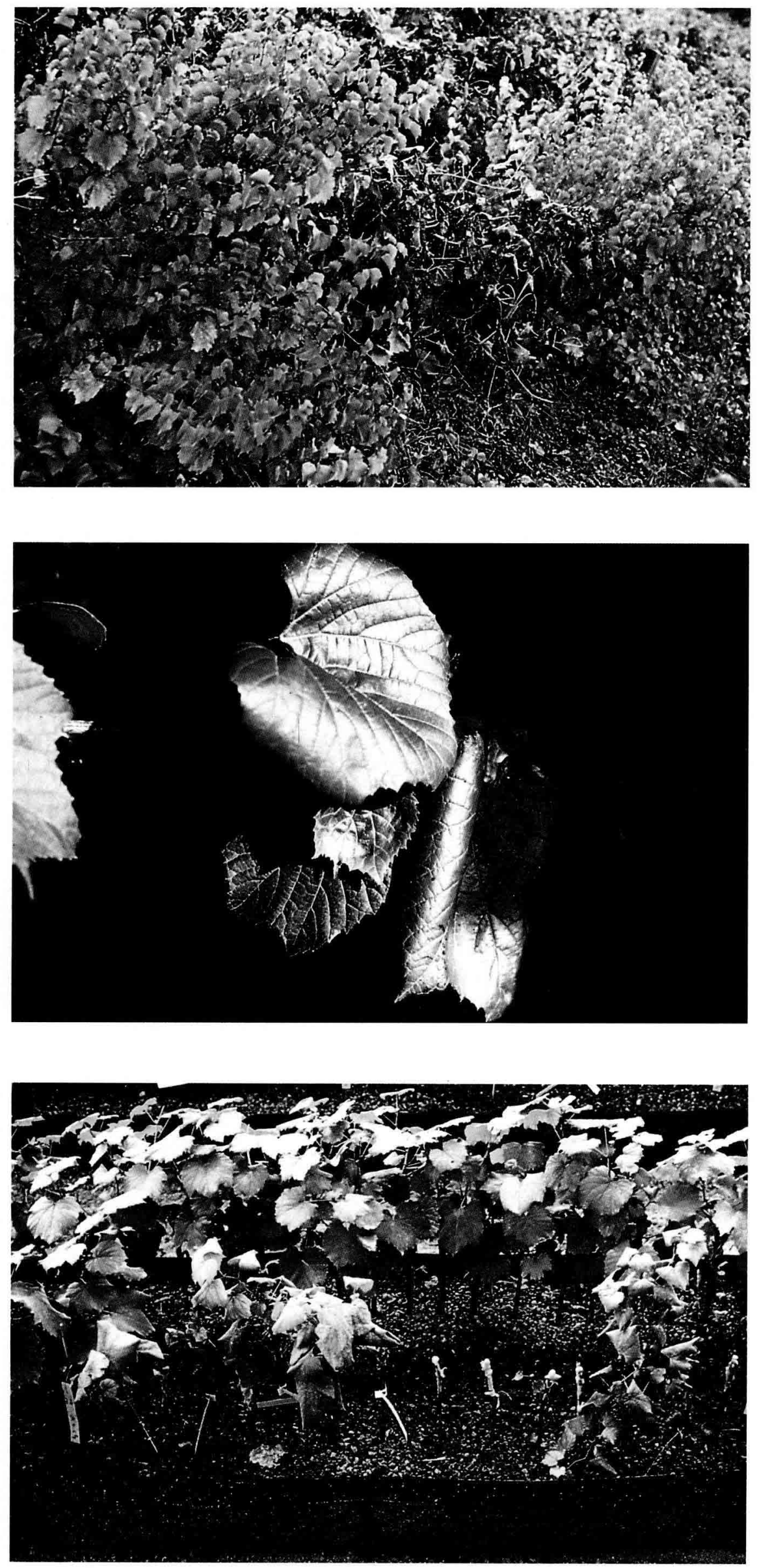

Fig 1. Repousses du porte-greffe $\checkmark$ rupestris cv Lot après la mort du greffon Nielluccio, suite à une épidémie de flavescence dorée (Corse, 1972). Le $V$ rupestris montre des feuilles uniformément jaunes et repliées en cuillère vers le dessus, indiquant ainsi une persistance pluriannuelle de l'infection. Au centre, un Nielluccio malade (FD) encore vivant se distingue par un rougissement prononcé du feuillage.

Fig 2. Symptôme de FD rencontré de façon exceptionnelle sur des repousses du porte-greffe SO4, à la base d'un cep malade de FD. Aucune autre repousse n'a montré de symptôme dans le reste du vignoble.

Fig 3. Indexage en serre du portegreffe $3309 \mathrm{C}$ avec la variété sensible Chardonnay. Les greffes malades (FD) se suivent fréquemment en laissant entre elles des séries de morts, souvent dus, eux aussi, à la FD. La mortalité totale (FD + morts) due à la maladie peut atteindre $82 \%$ chez certaines descendances de ceps de $3309 \mathrm{C}$ sans symptôme. 


\section{Les ceps de 3309 C classés $A$}

Les tableaux I, II et III présentent les caractéristiques et l'évolution des ceps de classe A, repérés respectivement en 1988, 1989 et 1990.

Les premières observations, faites en 1988, ont permis de repérer 10 ceps de classe A. Ce sont les seuls pour lesquels nous disposons de 3 ans de notations et d'indexages 1989, 1990 et 1991 (tableau I). Sur ces 10 ceps, 4 seulement se sont révélés positifs à l'indexage, c'est-à-dire aptes à transmettre la FD par la greffe. Ils ont transmis en 1989 la maladie dans une forte proportion de leurs boutures vivantes, allant de 36 à $100 \%$. Les $100 \%$ ont été trouvés sur un faible effectif où la précision du résultat est très faible. II faut retenir que l'infectivité n'est pas généralisée sur les ceps malades.

Cette forte transmission de la FD par indexage est associée à une forte mortalité des greffes (tableau I, 1989). Il peut être admis qu'une part importante de cette mortalité doit être imputée à la FD (fig 3 ).

La suite des notations de ces 4 ceps au champ, en 1990 et 1991 a montré soit une permanence de la note $A$, soit une évolution vers la mort du cep en 1 ou 2 ans. Les indexages se retrouvent positifs dans les années successives, à l'exception d'un cep négatif en 1990, mais de nouveau positif en 1991.
Les mêmes observations peuvent être faites pour les ceps de classe A qui se sont révélés en 1989 (tableau II) et en 1990 (tableau III). Une partie seulement des ceps classés $A$ se révèle positive $F D$ en indexage: 4/10 en 1988 (tableau I), 11/15 en 1989 (tableau II) et 29/45 en 1990 (tableau III).

Les ceps de classe A, positifs en indexage, transmettent toujours la FD par greffage dans une forte proportion, associée à une forte mortalité des greffes (tableaux I, II, III).

L'évolution des "symptômes" montre soit une permanence de la note $A$, soit une évolution vers la mort. Nous noterons cependant que l'évolution vers la mort se réduit progressivement du tableau I au tableau III, ce qui peut être l'indice que les premiers ceps repérés en classe A (tableau I) pourraient avoir un problème sanitaire supplémentaire.

L'examen des ceps classés $A$, négatifs à I'indexage (bas des tableaux I, II et III) a montré une faible mortalité au greffage. Leur évolution a été diverse, soit un retour à la normale, soit le maintien en note $A$, soit un passage en note $B$.

\section{Les ceps de 3309 classés B}

Les ceps de classe B repérés en 1989 et 1990 sont présentés respectivement dans les tableaux IV et $\mathrm{V}$.

Nous voyons immédiatement que la proportion de ceps de classe $B$, révélée positive

Tableau I. Notations et indexages successifs des ceps de $3309 \mathrm{C}$ repérés de classe A en 1988 au champ.

\begin{tabular}{|c|c|c|c|c|c|c|c|c|c|c|c|c|c|c|c|}
\hline & \multicolumn{5}{|c|}{ Notations 1989} & \multicolumn{5}{|c|}{ Notations 1990} & \multicolumn{5}{|c|}{ Notations 1991} \\
\hline & \multicolumn{2}{|c|}{ Indexage } & \multicolumn{3}{|r|}{ Champ } & \multicolumn{3}{|c|}{ Indexage } & \multirow{2}{*}{\multicolumn{2}{|c|}{$\begin{array}{l}\text { Champ } \\
\% \text { FD/ Note } \\
\text { Viv Nol }\end{array}$}} & \multirow[b]{2}{*}{ Total } & \multicolumn{2}{|c|}{ Indexage } & \multirow{2}{*}{\multicolumn{2}{|c|}{$\begin{array}{l}\text { Champ } \\
\% \text { FD/ Note } \\
\text { Viv Nol }\end{array}$}} \\
\hline & Total & Mort & $F D$ & $\begin{array}{c}\% \text { FD/ } \\
\text { Viv }\end{array}$ & Note & Total & Mort & $F D$ & & & & Mort & $F D$ & & \\
\hline 4 ceps & 80 & 46 & 17 & 50 & $A$ & 46 & 4 & 34 & 81 & + & & & & & \\
\hline indexés & 14 & 12 & 2 & 100 & $A$ & 5 & 0 & 2 & 40 & + & & & & & \\
\hline FD & 27 & 22 & 2 & 40 & $A$ & 19 & 0 & 0 & 0 & $A$ & 23 & 10 & 6 & 46 & $A$ \\
\hline en 1989 & 78 & 53 & 9 & 36 & + & & & & & & & & & & \\
\hline $\begin{array}{l}6 \text { ceps } \\
\text { indexés } \\
\text { négatifs }\end{array}$ & & & & & & & & & & $\mathrm{N}$ & & & & & \\
\hline
\end{tabular}

FD: malade, $N$ : normal ; + : mort. Nous indiquons, pour chaque année de notation, 1989, 1990 et 1991, et pour chaque cep indexé, le total des boutures indexées par la greffe (Total), le nombre de greffes mortes (Mort), le nombre de greffes FD (FD) et le pourcentage de greffes FD, rapporté au nombre de greffes vivantes (\% FD/Viv). Enfin, nous indiquons la note $(A, B, C$ ou $N)$ donnée dans la même année sur le pied mère au champ. 
Tableau II. Notations et indexages successifs des ceps de 3309 C repérés de classe A en 1989 au champ.

\begin{tabular}{|c|c|c|c|c|c|c|c|c|c|c|}
\hline & & Notati & 1990 & & & & & ions & 991 & \\
\hline & & dexag & & & Champ & & Ind & & & Champ \\
\hline & Total & Mort & $F D$ & $\% F D / V i v$ & Note & Total & Mort & $F D$ & $\% F D / V i v$ & Note \\
\hline 11 ceps & 25 & 11 & 7 & 50 & A & 21 & 13 & 2 & 25 & A \\
\hline indexés & 66 & 14 & 41 & 79 & + & & & & & \\
\hline FD & 28 & 8 & 15 & 75 & $A$ & 14 & 13 & 0 & 0 & + \\
\hline en 1990 & 11 & 1 & 4 & 40 & A & 33 & 17 & 5 & 31 & + \\
\hline & 8 & 3 & 4 & 80 & $A$ & 37 & 10 & 2 & 7 & $A$ \\
\hline & 47 & 9 & 17 & 45 & + & & & & & \\
\hline & 12 & 3 & 3 & 33 & $A$ & 17 & 14 & 0 & 0 & A \\
\hline & 8 & 1 & 4 & 57 & $A$ & 5 & 4 & 0 & 0 & $A$ \\
\hline & 8 & 2 & 2 & 33 & + & & & & & \\
\hline & 12 & 2 & 6 & 60 & $A$ & 10 & 2 & 1 & 13 & $A$ \\
\hline & 6 & 2 & 4 & 100 & + & & & & & \\
\hline
\end{tabular}

4 ceps indexés négatifs

Légende: voir tableau I.

FD en indexage, devient beaucoup plus faible que dans la classe $A: 5 / 29$ pour les $B$ de 1989 (tableau IV) et 4/94 pour les B de 1990 (tableau V). La notation en classe B est donc beaucoup moins sûre pour la $F D$ que la classe $A$.

Les ceps de classe B, positifs en indexage, transmettent la FD dans une faible proportion, variant de 13 à $40 \%$, beaucoup plus faible que les $A$. Ils se révèlent moins malades que les ceps positifs de classe $A$, mais aussi plus dangereux parce que moins repérables. De même, la mortalité des greffes n'a pas été forte dans ces $B$ positifs en indexage (tableaux IV et V). Les symptômes de ces B positifs semblent montrer une tendance à l'aggravation vers la note $A$ dès l'année suivante (tableaux IV et $\mathrm{V}$ ).

Les ceps notés en classe $B$, mais restés négatifs en indexage, sont très nombreux (bas des tableaux IV et $\mathrm{V}$ ). Une majorité d'entre eux s'est maintenue en note $B$ l'année suivante ou a évolué vers la normale $\mathrm{N}$. Le classement en $B$ constitue donc un mauvais repérage des ceps malades de $3309 \mathrm{C}$.

\section{Les ceps de 3309 classés C}

Les 37 ceps classés $C$ retenus en 1989 sont présentés dans le tableau VI. Deux seulement se sont révélés positifs en indexage. Cette proportion est plus faible que dans les
$B$ et beaucoup plus faible que dans les $A$. Cela montre que la notation en classes $A, B$ et $C$ représente une approche intéressante du repérage visuel de la FD chez le portegreffe 3309. Ces 2 ceps ont transmis la FD dans une très faible proportion, de 10 à $11 \%$ plus faible que ceux classés $B$ et surtout que les A. La mortalité des greffes chez les $C$ indexés positivement a été faible $(2 / 21)$. Ces 2 ceps sans symptôme, classe $C$ mais indexés positifs, ont évolué vers la note $A$ en 1990 et 1991 et vers une capacité accrue de transmission de la FD en 1991 (37\%).

Les 35 ceps classés $C$, mais restés négatifs en indexage (bas du tableau VI) sont, dans leur majorité, restés en note $C$ ou passés en note $B$, mais en conservant un indexage négatif. Nous avons noté sur le tableau VI un cep de cette classe passé en note $A$ avec un indexage très positif $F D$ en 1991. Ce cas exceptionnel pourrait représenter une nouvelle inoculation, survenue malgré la couverture en insecticides.

\section{ÉTUDE DU CHAMP DE PIEDS MÈRES EXPÉRIMENTAL DE FERCAL}

L'étude du porte-greffes Fercal situé également dans l'Aude, dans la zone atteinte par l'épidémie de FD, a permis de retrouver les mêmes indices de cette maladie que pour le 
Tableau III. Notations et indexages des ceps de 3309 C repérés de classe A en 1990 au champ.

\begin{tabular}{|c|c|c|c|c|c|}
\hline & \multicolumn{3}{|c|}{ Notations 1991} & \multicolumn{2}{|r|}{ Champ } \\
\hline & Total & Mort & $F D$ & $\begin{array}{c}\% F D / \\
V i V\end{array}$ & Note \\
\hline 29 ceps & 32 & 17 & 6 & 40 & $A$ \\
\hline indexés & 16 & 8 & 4 & 50 & $A$ \\
\hline FD & 27 & 14 & 10 & 77 & + \\
\hline \multirow[t]{26}{*}{ en 1991} & 31 & 17 & 4 & 29 & $A$ \\
\hline & 30 & 18 & 4 & 33 & $A$ \\
\hline & 22 & 8 & 3 & 21 & $A$ \\
\hline & 35 & 12 & 9 & 39 & $A$ \\
\hline & 16 & 6 & 2 & 20 & $A$ \\
\hline & 30 & 14 & 2 & 13 & $A$ \\
\hline & 12 & 4 & 1 & 13 & $A$ \\
\hline & 21 & 11 & 2 & 20 & $A$ \\
\hline & 13 & 7 & 4 & 67 & A \\
\hline & 10 & 6 & 1 & 25 & A \\
\hline & 6 & 4 & 2 & 100 & $A$ \\
\hline & 8 & 6 & 1 & 50 & B \\
\hline & 17 & 9 & 4 & 50 & $A$ \\
\hline & 22 & 16 & 4 & 67 & A \\
\hline & 14 & 13 & 1 & 100 & + \\
\hline & 20 & 15 & 4 & 80 & $A$ \\
\hline & 12 & 5 & 2 & 29 & $A$ \\
\hline & 27 & 10 & 2 & 12 & $A$ \\
\hline & 14 & 5 & 3 & 33 & $A$ \\
\hline & 9 & 8 & 1 & 100 & $A$ \\
\hline & 11 & 9 & 1 & 50 & $A$ \\
\hline & 9 & 2 & 1 & 14 & A \\
\hline & 9 & 7 & 2 & 100 & $A$ \\
\hline & 23 & 8 & 9 & 60 & $A$ \\
\hline & 20 & 7 & 4 & 31 & $A$ \\
\hline & 14 & 5 & 3 & 33 & $A$ \\
\hline
\end{tabular}

16 ceps indexés négatifs

Légende: voir tableau $\mathrm{I}$.
Tableau V. Notations et indexages des ceps de 3309 C repérés de classe $B$ en 1990 au champ.

\begin{tabular}{|c|c|c|c|c|c|}
\hline & \multicolumn{4}{|c|}{$\begin{array}{l}\text { Notations } 1991 \\
\text { Indexage }\end{array}$} & \multirow{2}{*}{$\begin{array}{c}\text { Champ } \\
\text { Note }\end{array}$} \\
\hline & Total & Mort & $F D$ & $\begin{array}{c}\% F D / V i \\
v\end{array}$ & \\
\hline 4 ceps & 25 & 8 & 1 & 6 & $A$ \\
\hline indexés & 25 & 11 & 5 & 36 & A \\
\hline FD & 23 & 9 & 2 & 14 & $A$ \\
\hline en 1991 & 5 & 4 & 1 & 100 & $A$ \\
\hline
\end{tabular}

Légende : voir tableau 1.

3309 C. Les mêmes classes A, B et C ont été différenciées visuellement. L'analyse a été commencée en 1990 et poursuivie en 1991.

\section{Les ceps de Fercal repérés en classe $A$}

Quinze ceps de Fercal ont été repérés en classe A (tableau VII). Le Fercal ayant eu une mauvaise reprise au greffage, les indexages de l'hiver 1990-1991 ont été remplacés par des greffes en vert. Ces indexages en vert n'ont pu être réalisés que sur des effectifs réduits, mais ils ont donné de bons résultats. Sur les 15 ceps rattachés à la classe $A, 5(33 \%)$ se sont révélés positifs dans cet indexage en vert (tableau VII).

Ces ceps de classe $A$, positifs en indexage, sont restés en note A en 1991, à une exception près.

Tableau IV. Notations et indexages successifs des ceps de 3309 C repérés de classe B en 1989 au champ.

\begin{tabular}{|c|c|c|c|c|c|c|c|c|c|c|}
\hline & & Votatio & 199 & & & & Not & ions & 1991 & \\
\hline & & exage & & & Champ & & Index & & & Champ \\
\hline & Total & Mort & $F D$ & $\%$ FD/Viv & Note & Total & Mort & $F D$ & $\%$ FD/Viv & Note \\
\hline 5 ceps & 13 & 0 & 5 & 38 & $A$ & 1 & 1 & 0 & & A \\
\hline indexés & 13 & 3 & 2 & 20 & + & & & & & \\
\hline FD & 11 & 3 & 1 & 13 & $B$ & 15 & 5 & 0 & 0 & + \\
\hline en & 6 & 1 & 1 & 20 & A & & & & & $A$ \\
\hline 1990 & 6 & 1 & 2 & 40 & A & 25 & 11 & 6 & 43 & A \\
\hline
\end{tabular}

Légende: voir tableau 1. 
Tableau VI. Notations et indexages successifs des ceps de 3309 C repérés de classe C en 1989.

\begin{tabular}{|c|c|c|c|c|c|c|c|c|c|c|}
\hline \multicolumn{6}{|c|}{ Notations 1990} & \multicolumn{5}{|c|}{ Notations 1991} \\
\hline & \multicolumn{3}{|c|}{ Indexage } & \multicolumn{2}{|r|}{ Champ } & \multicolumn{3}{|c|}{ Indexage } & \multicolumn{2}{|r|}{ Champ } \\
\hline & Total & Mort & $F D$ & $\%$ FDN Viv & Note & Total & Mort & $F D$ & $\%$ FD/Viv & Note \\
\hline 2 ceps & 12 & 2 & 1 & 10 & $A$ & 26 & 18 & 3 & 37 & $A$ \\
\hline $\begin{array}{l}\text { Indexés } \\
\text { FD } \\
\text { en } 1990\end{array}$ & 9 & 0 & 1 & 11 & A & & & & & A \\
\hline $\begin{array}{l}35 \text { ceps } \\
\text { indexés } \\
\text { négatifs }\end{array}$ & & & & & & \multicolumn{5}{|c|}{$\begin{array}{l}1 \text { cep indexé FD } \\
\text { (avec } 83 \% \text { de greffes FD) }\end{array}$} \\
\hline
\end{tabular}

Légende: voir tableau 1.

Tableau VII. Notations et indexages des Fercal repérés de classe $A$ en 1990 .

\begin{tabular}{|c|c|c|c|c|}
\hline \multicolumn{5}{|c|}{ Notations 1991} \\
\hline & $\begin{array}{c}\text { Nb } \\
\text { greffes } \\
\text { en vert }\end{array}$ & $\begin{array}{c}\text { Greffons } \\
\text { FD }\end{array}$ & $\begin{array}{c}\% \\
\text { FD/Viv }\end{array}$ & $\begin{array}{c}\text { Champ } \\
\text { Note }\end{array}$ \\
\hline 5 ceps & 4 & 1 & 25 & $A$ \\
\hline indexés & 5 & 1 & 20 & $B$ \\
\hline FD & 4 & 1 & 25 & + \\
\hline en 1991 & 2 & 1 & 50 & $A$ \\
\hline & 4 & 2 & 50 & $A$ \\
\hline
\end{tabular}

10 ceps indexés négatifs

Légende: voir tableau I. La reprise au greffage d'hiver n'ayant pas été bonne, l'indexage a été repris en greffe en vert en utilisant comme indicateurs le Chardonnay et le Pinot noir. Pour simplifier la présentation, nous ne séparons pas les résultats de ces 2 indicateurs.

Les 10 ceps de classe $A$, restés négatifs en indexage et donc sains, sont également restés $A$ en 1991.

\section{Les ceps de Fercal repérés en classe B}

Huit ceps de Fercal ont été repérés en classe B. Ils ont été indexés en greffe en vert, mais aucun ne s'est révélé FD positif.

\section{ÉTUDE DE 8 AUTRES VARIÉTÉS DE PORTE-GREFFES}

Confrontés à l'importance du travail que représente l'indexage de tout un champ de pieds mères, nous avons, ici encore, tenté de percevoir des signes de maladie chez 8 autres variétés de porte-greffes, apparemment sans symptôme.

Les variétés 110 Richter, 140 Ruggeri, 101.14 Millardet et 125 AA Téléki ont montré des retards de végétation au printemps et quelques non-aoûtements à l'automne, moins marqués que dans le cas du $3309 \mathrm{C}$ ou du Fercal. Cependant aucun des ceps classés A ou B dans ces 4 variétés n'a été positif en indexage.

Les variétés SO4, 161-49 Couderc et Kober 5.BB montrent occasionnellement des retards de végétation au printemps, mais aucun signe de non-aoûtement. Le porte-greffe 41.B a été particulièrement étudié, mais aucun symptôme n'a pu être relevé. L'indexage n'a donc pas été entrepris sur ces 4 variétés.

\section{SITUATIONS DES BOUTURES INFECTIEUSES SUR LES RAMEAUX DES CEPS DE 3309 C}

Nous avons vu que les ceps malades de $3309 \mathrm{C}$ ne montraient jamais leur infectivité sur la totalité de leurs boutures. Les ceps de 3309 C classés A transmettent la FD dans une forte proportion et ceux classés $B$, dans une plus faible proportion. II se posait donc la question de savoir où se trouvaient les boutures infectieuses le long du rameau et éventuellement sur quels rameaux.

Dans ce but, 102 rameaux entiers ont été récoltés durant l'hiver 1990-1991. Les boutures ont été greffées en greffe d'hiver avec du Chardonnay, puis plantées dans l'ordre où elles étaient sur le rameau, en les numérotant à partir de la base.

Le tableau VIII présente les résultats sur 45 rameaux de ceps de classe $A$ et le tableau IX sur 10 rameaux de ceps de classe $B$. Cha- 
Tableau VIII. Situation des boutures infectieuses sur les rameaux de ceps malades de $3309 \mathrm{C}$ (ceps de classe A).

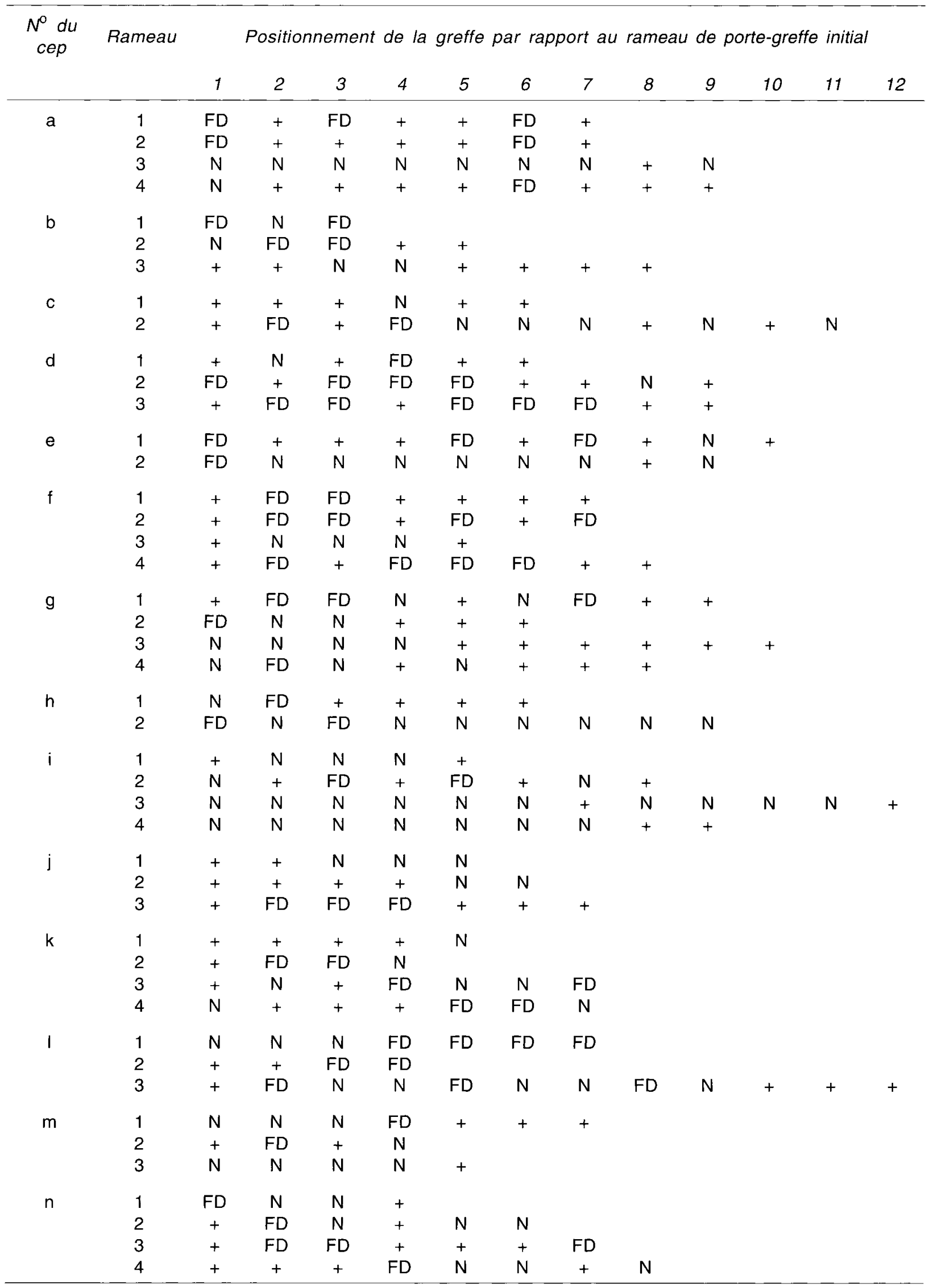

La base du rameau est à gauche (bouture 1). FD: bouture indexée flavescence dorée; $N$ : bouture indexée normale (non FD) ; +: greffe morte. 
Tableau IX. Situation des boutures infectieuses sur les rameaux de ceps malades de $3309 \mathrm{C}$ (ceps de classe B).

\begin{tabular}{|c|c|c|c|c|c|c|c|c|c|c|c|c|c|}
\hline \multirow[t]{2}{*}{$\begin{array}{c}N^{\circ} d u \\
c e p\end{array}$} & \multirow[t]{2}{*}{ Rameau } & \multicolumn{4}{|c|}{ Positionnement de la } & \multirow{2}{*}{$\begin{array}{c}\text { greffe } \\
5\end{array}$} & \multicolumn{2}{|c|}{ par rapport al } & \multicolumn{4}{|c|}{ rameau de porte-greffe initic } & \multirow[b]{2}{*}{12} \\
\hline & & 1 & 2 & 3 & 4 & & 6 & 7 & 8 & 9 & 10 & 11 & \\
\hline \multirow[t]{4}{*}{0} & 1 & + & $\mathrm{N}$ & $N$ & $N$ & $\mathrm{~N}$ & & & & & & & \\
\hline & 2 & FD & + & + & + & $N$ & $N$ & & & & & & \\
\hline & 3 & $\mathrm{~N}$ & $\mathrm{~N}$ & $N$ & $N$ & $N$ & $N$ & & & & & & \\
\hline & 4 & + & + & + & + & $N$ & $N$ & $N$ & + & & & & \\
\hline \multirow[t]{3}{*}{$\mathrm{p}$} & 1 & FD & + & + & $N$ & $N$ & + & & & & & & \\
\hline & 2 & FD & FD & + & + & + & FD & + & $\mathrm{N}$ & & & & \\
\hline & 3 & + & FD & $N$ & $\mathrm{~N}$ & $\mathrm{~N}$ & $\mathrm{~N}$ & & & & & & \\
\hline \multirow[t]{3}{*}{$q$} & 1 & $N$ & $\mathrm{~N}$ & $N$ & $\mathrm{~N}$ & + & + & & & & & & \\
\hline & 2 & $\mathrm{~N}$ & $N$ & $\mathrm{~N}$ & $\mathrm{~N}$ & FD & $N$ & $\mathrm{FD}$ & & & & & \\
\hline & 3 & + & + & $\mathrm{N}$ & $\mathrm{N}$ & $N$ & + & & & & & & \\
\hline
\end{tabular}

FD: bouture indexée flavescence dorée; $N$ : bouture indexée normale (non FD); + : greffe morte.

que cep est représenté par 2 à 4 rameaux. Ces tableaux ne présentent donc qu'un échantillon des 102 rameaux étudiés.

II apparaît que pour un même cep, les différents rameaux ne sont pas comparables. Ainsi, le cep a montre 3 rameaux avec des boutures indexées FD et un rameau entièrement normal $\mathrm{N}$.

En second lieu, un même rameau présente souvent à la fois des boutures indexées FD et des boutures $N$ (par exemple : cep $c$ - rameau 2).

Enfin, les boutures indexées FD ont une forte tendance à se retrouver groupées ou entourées de greffes mortes. Cela suggère fortement que les zones infectées des sarments englobent un certain nombre de boutures successives. Cette dernière observation nous incite à évaluer l'étendue des zones infectieuses sur les sarments.

\section{Étendue des zones infectieuses sur les sarments}

Pour évaluer l'étendue des zones infectieuses le long des sarments, nous avons multiplié la longueur d'une bouture $(30 \mathrm{~cm})$ par le nombre de boutures FD contiguës. $\mathrm{Ce}$ pendant les boutures des extrémités qui se révèlent FD ne sont pas forcément infectées au départ dans la totalité de leur longueur. Une telle bouture peut avoir une fraction de centimètre infectée, quelques $\mathrm{cm}$ ou les $30 \mathrm{~cm}$. Nous avons donc convenu de compter l'infection des boutures FD des ex- trémités pour la moitié de leur longueur, soit $15 \mathrm{~cm}$. Cela réduit l'erreur au minimum pour les boutures des extrémités.

Le calcul est également rendu aléatoire par la présence de nombreuses greffes mortes. Celles-ci sont souvent mortes du fait de la FD, mais rien ne peut être assuré dans chaque cas particulier. Nous pouvons cependant admettre qu'une greffe morte au milieu d'une suite de FD peut être considérée comme FD (fig 3).

Dans ces conditions, l'examen des 102 rameaux (dont les tableaux VIII et IX ne donnent qu'un échantillon) permet d'estimer que les zones infectieuses s'étendent sur une longueur de 15 à $150 \mathrm{~cm}$ (cep g, rameau 2 et 4 comparés au cep $d$, rameau 3 ).

\section{Situation des zones infectieuses le long des rameaux}

Les profils des 102 rameaux étudiés ont été additionnés de façon à voir les effectifs de greffes FD ou $\mathrm{N}$ qui apparaissent aux différents niveaux du rameau (fig 4).

Nous avons testé sur ces effectifs l'hypothèse selon laquelle les greffes FD apparaîtraient au hasard à un niveau quelconque du rameau. Selon le test statistique $\chi^{2}$, nous pouvons admettre l'hypothèse de l'homogénéité ( $P_{\chi^{2}}=0,50$ avec 6 degrés de liberté). II n'y aurait donc pas de différence significative entre les différents niveaux des rameaux. 


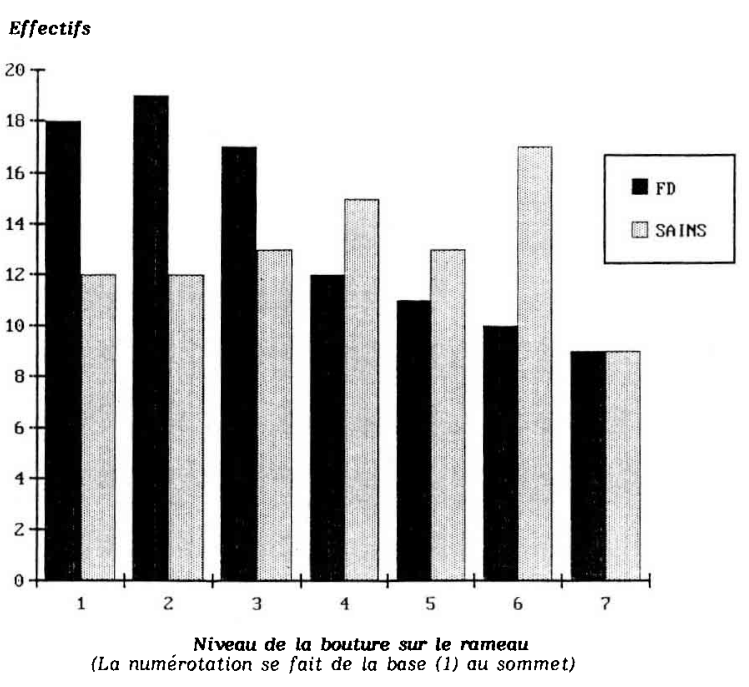

Fig 4. Situation des zones infectieuses le long des rameaux du porte-greffe $3309 \mathrm{C}$. Ce graphique représente les effectifs cumulés des différents niveaux des 102 rameaux étudiés.

\section{CONCLUSIONS ET DISCUSSION}

La première conclusion que nous pouvons tirer de ce travail est que beaucoup de variétés de porte-greffes, en repousses sur des ceps malades ou en champs de pieds-mères, sont porteuses sans symptôme de la flavescence dorée. Quelques symptômes sont parfois reconnaissables chez certaines variétés: Rupestris du Lot, 420 A, SO4, 1103 Paulsen, mais ils restent sporadiques et ne peuvent en aucun cas servir à repérer la totalité des ceps porteurs.

Faute de pouvoir repérer la FD sur le porte-greffe par son syndrome classique, nous nous sommes attachés à 2 caractères: le retard de végétation au printemps et le mauvais aoûtement de certains rameaux, pour identifier les ceps malades dans les champs de pieds mères d'un certain nombre de variétés de porte-greffes. Cette association de 2 symptômes (classe A) a permis de repérer les ceps malades dans 40 à $73 \%$ des cas chez le $3309 \mathrm{C}$ et dans $33 \%$ des cas chez le Fercal. Ces 2 variétés doivent donc être considérées comme porteuses potentielles de la FD. En revanche, cette association de symptômes n'est pas apparue comme signe de FD pour les autres variétés étudiées, 110 Richter, 140 Ruggeri, 101.14 MG, 125 AA, SO4, 161-49 Couderc, Kober 5 BB et $41 \mathrm{~B}$.
La FD est transmise par des ceps de 3309 qui montrent des symptômes discrets de maladie, classe A ou classe B, mais aussi par des $3309 \mathrm{C}$ qui ne montrent aucun symptôme de maladie, classe $\mathrm{C}$ (tableau VI).

L'étude de l'évolution de ces ceps sur plusieurs années montre que tous les ceps indexés positifs sont porteurs de façon persistante de la FD, avec semble-t-il une tendance à l'aggravation (tableaux IV, $\mathrm{V}$ et VI). Cela signifie que, si les traitements insecticides empêchent l'infection de nouveaux ceps, ils n'empêchent pas les ceps déjà atteints de produire des boutures infectieuses, et cela pendant longtemps. Ce comportement très virologique diffère de celui de la plupart des variétés de $V$ vinifera, chez lesquelles on observe un rétablissement qui se maintient en l'absence de réinoculations par l'insecte vecteur (Caudwell, 1964).

L'analyse des descendances des ceps porteurs montre que les ceps de $3309 \mathrm{C}$ indexés positifs pour la FD ne transmettent cette maladie que par une partie de leur boutures (tableaux ( à VI). La FD n'est pas généralisée sur les ceps malades. Cela pose des problèmes pour les échantillonnages des épreuves de diagnostic, car les tests sérologiques devront obligatoirement être faits sur un échantillonnage important. Puisque par ailleurs la concentration en antigènes est toujours faible chez les porte-greffes (Caudwell et Kuszala, 1992), ces considérations obligent à recourir à des tests d'une très grande sensibilité, tels que le test génomique PCR qui a été mis au point dans notre laboratoire (Daire et al, 1993).

Les boutures infectieuses sont réparties au hasard le long du rameau (tableaux VIII et IX ; fig 4), en des zones malades dont l'étendue varie de quelques centimètres à $150 \mathrm{~cm}$. On s'interroge sur la cause de cette répartition. De toute évidence, l'infection persiste chez les pieds de 3309 malades et elle ne peut se maintenir en hiver que dans la souche rasée qui reste après la taille. Pourquoi, dès lors, les bois nouveaux ne sont-ils pas tous infectés régulièrement sur toute leur longueur? Quels sont les facteurs biologiques ou physiques apparemment fortuits qui président au caractère normal $(N)$ ou infectieux (FD) des boutures successives? $\mathrm{Ce}$ problème mérite d'être étudié comme révélateur d'une méthode de contrôle de cette maladie. II doit être mis en comparaison avec 
les phénomènes de rétablissement décrits précédemment sur les variétés de $V$ vinifera et sur certains de leurs hybrides, tels que le Baco 22 A (Caudwell, 1964). II faudra toutefois garder à l'esprit que, dans ce dernier cas, les "symptômes localisés " étaient provoqués par des réinoculations dont les effets se trouvaient confinés par une réaction de défense propre à ces variétés de vigne. Cette cause des symptômes localisés ne peut être retenue ici pour ces porte-greffes qui ne sont pas réinoculés.

La forte transmission de la FD par indexage des 3309 C classés A est associée à une forte mortalité des greffes (tableaux I, II et III). Si nous voulons nous placer du point de vue économique du pépiniériste, nous devons parler d'une "mortalité pratique» qui serait la somme des greffes mortes et des greffes FD qui ne survivent que rarement à l'année de pépinière. Cette mortalité pratique devient très élevée, atteignant dans le tableau I, 163 boutures mortes ou devant mourir sur un total de 199 , soit $82 \%$. On compte de même $70 \%$ de mortalité pratique dans le tableau II, et $69 \%$ dans le tableau III. Cette "mortalité pratique » est économiquement intolérable.

Le caractère normal, c'est-à-dire sans symptôme d'une greffe n'établit pas un état sanitaire sain. Un essai est mis en place pour voir le devenir des boutures n'ayant pas transmis la FD en première année et évaluer les risques de propagation de la maladie par les greffes sans symptôme.

II faut aussi relever l'extrême hétérogénéité des ceps pieds-mères de $3309 \mathrm{C}$ infectés. Les ceps classés A indexés malades ont transmis la FD dans une très forte proportion. Mais on a relevé également des ceps classés $B$ qui transmettent la FD dans une faible proportion. Enfin, certains ceps sans symptôme apparent, classe $C$, transmettent aussi la FD. On s'interroge sur les causes de cette hétérogénéité dans une plantation clonale, sur des ceps qui ont été infectés de la même manière et presque simultanément.

II y a aussi hétérogénéité pour l'évolution des ceps malades vers la mort; très forte dans le tableau I, puis de moins en moins forte en allant vers le tableau II puis vers le tableau III. Nous avons pensé que, dans la première notation (tableau I), des ceps pouvant être atteints d'un autre désordre pathologique avaient pu être englobés dans la classe A. L'existence de certains ceps de classe $A$, négatifs à l'indexage mais évoluant vers la mort, pourrait renforcer cette interprétation. Nous nous sommes assurés que l'eutypiose n'interférait en rien dans ces notations (Dubos, INRA Bordeaux).

II faut maintenant revenir sur le cep de $3309 \mathrm{C}$ de classe $C$, négatif en indexage en 1990 , passé en note $A$, avec un indexage très positif l'année suivante (tableau VI). Ce cas exceptionnel pourrait représenter une nouvelle inoculation, malgré la couverture insecticide. II souligne l'importance d'un isolement géographique des champs de pieds mères, que ne remplace pas la couverture insecticide. Cette exigence d'isolement a été plusieurs fois soulignée dès la reprise de l'épidémie de FD dans le sud de la France et en Italie du Nord (Caudwell, 1989).

Ce travail, basé sur la présence d'indices de maladie chez le $3309 \mathrm{C}$ et le Fercal, n'a pu être étendu aux autres variétés de portegreffes qui ne montrent aucun symptôme. La présente méthode nous aurait obligés à opérer des prélèvements au hasard dans la parcelle, ce qui est trop aléatoire pour un travail d'indexage. Un protocole d'inoculation contrôlée est donc mis en place pour ces variétés.

\section{RÉFÉRENCES}

Caudwell A (1964) Identification d'une nouvelle maladie à virus de la vigne, la aflavescence dorée". Étude des phénomènes de localisation des symptômes et de rétablissement. Ann Epiphyties, vol 15, hors série 1, $193 \mathrm{p}$

Caudwell A (1989) La flavescence dorée et la multiplication du matériel végétal vigne. Le pépiniériste 64, 9-13

Caudwell A, Kuszala C (1992) Mise au point d'un test Elisa sur les tissus de vignes atteintes de flavescence dorée. Res Microbiol 143, 791-806

Caudwell A, Moutous G, Brun P, Larrue J, Fos A, Blancon G, Schick JP (1974) Les épidémies de flavescence dorée en Armagnac et en Corse et les nouvelles perspectives de lutte contre le vecteur par des traitements ovicides d'hiver. Bull Tech Inf 294, 783-794

Daire X, Clair D, Larrue J, Boudon-Padieu E, Caudwell A (1993) Diversity among Mycoplasma-like organisms inducing grapevine yellows in France. Vitis 32, 159-163

Moutous G (1977) Définition des symptômes de la flavescence dorée sur les variétés de portegreffes. Rev Zoo Agric 76, 90-98 